\title{
Buck-Boost DC-DC Converter Control by Using the Extracted Model from Signal Flow Graph Method
}

\author{
Leila Mohammadian, Ebrahim Babaei*1, Mohammad Bagher Bannae Sharifian
}

Accepted 22 $2^{\text {th }}$ March 2015

DOI: 10.18100/ijamec.04927

\begin{abstract}
In this paper, the signal flow graph technique and Mason gain formula are applied for extracting the model and transfer functions from control to output and from input to output of a buck-boost converter. In order to investigate a controller necessity for the converter of assumed parameters, the frequency and time domain analysis are done and the open loop system characteristics are verified and the needed closed loop controlled system specifications are determined. Finally designing a controller for the mentioned converter system based on the extracted model is discussed. Then, a modern control design method is employed for regulator design. For this purpose, a full state feedback control for pole placement is applied. The simulation results are used to show the performance of the proposed modeling and regulation method.
\end{abstract}

Keywords: Buck-boost dc-dc converter, signal flow graph, full state feedback, model based controller.

\section{Introduction}

Recently, the different type of dc-dc converters such as buckboost converter are mostly used in industrial applications [1-2] This paper focuses on modelling this converter by means of signal flow graph technique and then analysing the obtained models of the converter is investigated. For achieving an effective controller for a dc-dc converter, a good and proper model of the converter is needed [3]. In order to get the frequency response of the converters to design a controller and compensator circuits, modelling of the system is inevitable. The model describes how control actions and disturbances are expected to affect the behaviour of the system. Previous works present different models for dc-dc converters. Regarding that the power electronic converters include nonlinear elements like switches and diodes, then modelling of them needs linearization. So, applying averaging and linearization techniques has a special importance. After linearization, the inside model of the system is extractable and therefore investigating the frequency response will be possible [4-6].

Modelling dc-dc converters by using small signal linearization and averaging techniques cause complex equations. Solving these equations for basic converters is not a problem, but for high order converters, dealing with them will be more difficult.

In [7], both nonlinear and average linear models for a dc-dc converter have been presented. In [8-9], in order to derive a mathematical model of a dc-dc converter and study the transient states of it, a combination of Laplace and Z-transforms is employed.

Although a converter including switches is a nonlinear system, but it can be decomposed to two linear circuits; one for on-state and the other for off-state of the switch. Then, these two linear circuits are illustrated by means of two signal flow graphs. Composition of the two sub-graphs using switching branches reaches to the graph of the whole converter. Switching branches are the nonlinear parts of the converters. Thus, modelling process is limited to the switching branches. In [10-15], a signal flow graph method has been presented for modelling a dc-dc

${ }^{1}$ Faculty of Electrical and Computer Engineering, University of Tabriz,

Tabriz, Iran

*Corresponding Author: Email: e-babaei@tabrizu.ac.ir converter. Then, by using the Mason gain formula any desired transfer function of the system might be extracted and can be used to design any proper controller.

The proposed technique in this paper, give greatly simplified mathematical and graphical representation of the systems based on the signal flow graph of the converter. These methods have great advantage because of simplicity and being capable of giving any desired transfer function of the system to design a controller for a determined variable. Furthermore, by using the proposed graphical method, the intended models can be extracted and can be used for surveying nonlinear and dynamic behaviour of switching converters.

The main contribution of this paper is to derive model of the converter by using the proposed signal flow graph method. Then, the results can be used in design a model- based controller for the buck-boost converter. In this process, applying the mentioned signal flow graph technique and Mason gain formula, the input to output, control to output transfer functions are obtained. Then, the frequency domain responses in form of Bode magnitude and phase diagrams are achieved and the proper full state feedback controller in form of a pole placement method is designed. The proposed controller uses the desired poles given by a filter prototype that is optimal with respect to an integral performance index and discusses how to select a weighting parameter that determines the closed-loop pole locations [16]. At last, the simulation results by using the described modelling and controlling methods are given to show the good performance and behaviour of the modelling and control methods.

\section{Review on DC-DC Buck-Boost Converter}

The output voltage of a buck-boost dc-dc converter can be more or less than the input voltage. The converter has two operating modes. The first mode is when the switch is on and the second one is for off state of the switch. In both operating modes, the switch and the diode would be complementary. The output voltage has the opposite polarity of the input voltage. So, the converter is also referred to as a reverser converter. Fig. 1(a) shows the power circuit of the buck-boost converter. In this figure, $v_{g}$ is the input voltage; $L, C, R$ and $R_{L}$ refer to the inductor, capacitor, load resistor and parasitic resistance of the 
inductor, respectively. $v_{L}$ and $v_{o}$ are the inductor voltage and the output voltage, respectively. $i_{g}, i_{L}$ and $i_{o}$ are the input current, inductor current and the output current, respectively. The equivalent circuits of the two mentioned operating modes of the converter are shown in Fig. 1(b) and 1(c).

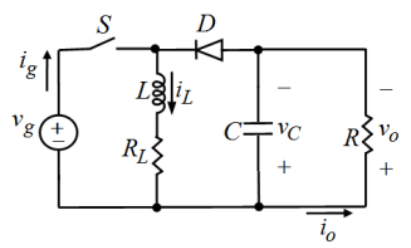

(a)

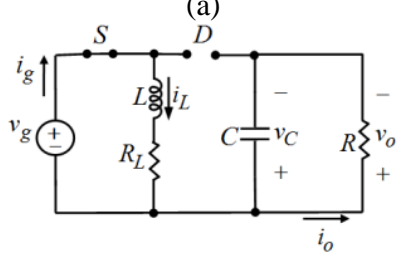

(b)

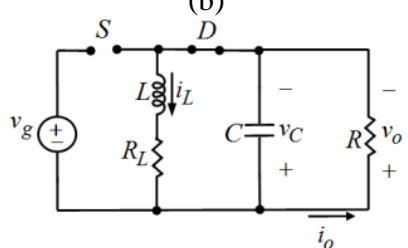

(c)

Figure 1. Buck-boost dc-dc converter; (a) the power circuit; (b) equivalent circuit in on-state; (c) equivalent circuit in off-state

\section{The Proposed Graph Modelling Method}

Before designing a controller for a system, the control system designer must know the system's characteristics. For example, is the open-loop system stable? Are there dominant poles? Are there poles that may be neglected during design? Is the system controllable by using the selected inputs? Can an estimator be designed based on the measured outputs? These types of questions should be answered before trying to design a controller for the system.

The nonlinear circuit of the converter is modelled as a smallsignal continuous linear time invariant (LTI) system by using averaging technique. The model is a mathematical description of the behaviour of the real system that is enough for performing stability test.

In order to model the converter by using the first method the below steps should be done:

- $\quad$ First, the state equations for both operating modes are extracted using Kireshof voltage and current laws.

- Then, the obtained state equations at the on time interval is multiplied in $\bar{d}_{1}$ and at the off time interval is multiplied in $1-\bar{d}_{1}=\bar{d}_{2}$, then they are added to each other. $\bar{d}_{1}$ is considered as the switch conduction coefficient and $\bar{d}_{2}$ as the switch non-conduction coefficient. $\bar{d}_{1}$ can range in 0 and 1 interval. The result is the averaged equations. Hence, the averaged equations are related to both on and off time intervals of the switch. In averaged equations, variables in form of $\bar{x}$ are average values which are different from the instantaneous variables in form of $x$.

- In averaged equations, $\mathrm{dc}$ and ac variables are substituted and rewritten with $\bar{X}$ and $\tilde{x}$, respectively. Considering all variables in form of $x=\bar{X}+\tilde{x}$ the small signal linearization is done. Regarding that the product of two de variables is a dc one and the product of one $\mathrm{dc}$ and one ac variable is an ac variable, also the product of two ac variables is zero, the obtained equations are rewritten.

- Finally, the extracted ac equations from the previous step are used to achieve the signal flow graph of the converter.

The whole state equations, the averaged and linearized form of them are given in Table 1. Model of the converter without the parasitic resistor of the inductor is extracted by using the proposed graphical method.

\subsection{Proposed Signal Flow Graph for the Converter}

AC small signal equations are used to plot the signal flow graph shown in Fig. 2. In these equations there are two variables; $\tilde{v}_{o}$ and $\tilde{i}_{L 1}$, and two inputs; $\tilde{d}$ and $\tilde{v}_{g}$. The graph is depicted for all independent variables $(s \tilde{x}, \tilde{x})$ by using nodes and paths between two nodes.

In order to extract transfer functions from signal flow graph, Mason gain formula $\left(\frac{y_{\text {out }}}{y_{\text {in }}}=\sum_{k=1}^{n} \frac{P_{k} \Delta_{k}}{\Delta}\right)$ will be used as given in the following.

For calculating transfer functions, the gain of distinct loops, nontouching loops, forward paths from each input to output are extracted as Table 2. The obtained transfer functions in existence and absence of the parasitic resistance of the inductor are given in Table 3.

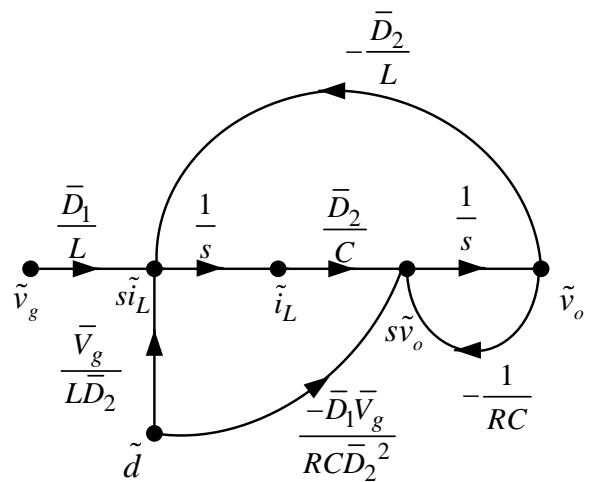

Figure 2. Signal flow graph for the buck-boost dc-dc converter by using the proposed method

\section{Simulation Results for Frequency Response of the Converter}

In order to survey the behaviour of the converter from stability point of view, a buck-boost dc-dc converter with the component values given Table 4 is considered. The root-locus diagrams for previously extracted transfer functions of the converter are shown in Fig. 3. The information obtained from these diagrams is given in Table 5. For analysis purpose the duty cycle of the converter is supposed to be 0.335 . Fig. 4 shows the magnitude and phase Bode diagrams.

From simulation results, it can be concluded that the system, $\tilde{v_{o}} / \tilde{d}$ is unstable and phase and gain margins for $\tilde{v_{o}} / \tilde{d}$ become; $G M=-21.6 d B$ and $P M=-61.2 \mathrm{deg}$. From root-locus diagrams, the mentioned system has a zero in right hand side of the imaginary margin. In order to have a more stable system the proper controller should be designed for the mentioned transfer function. In the next section, designing the proposed modelbased full state feedback controller will be explained. 
Table 1. Achieved equations from converter operating in two modes, averaged and linearized form of them State equations for Fig. 1(b)

$$
\begin{gathered}
L \frac{d i_{L}}{d t}=v_{g} \\
v_{C}=v_{o} \\
C \frac{d v_{O}}{d t}=-\frac{v_{O}}{R}
\end{gathered}
$$

State equations for Fig. 1(c)

The averaged equations for both modes

$$
\begin{gathered}
L \frac{d i_{L}}{d t}=v_{o} \\
v_{C}=v_{o} \\
C \frac{d v_{O}}{d t}=-\frac{v_{O}}{R}+i_{L}
\end{gathered}
$$

\begin{tabular}{|c|c|c|}
\hline Distinct loops & Distinct loops gain & Non-touching loops gain \\
\hline $\begin{array}{c}L p_{1}: s \tilde{i}_{L} \rightarrow \tilde{i}_{L} \rightarrow s \tilde{v}_{o} \rightarrow \tilde{v}_{o} \rightarrow s \tilde{i}_{L} \\
L p_{1}: s \tilde{v}_{o} \rightarrow \tilde{v}_{o} \rightarrow s \tilde{v}_{o}\end{array}$ & $\begin{array}{l}L p_{1}=-\frac{\bar{D}_{2}^{2}}{L C s^{2}} \\
L p_{2}=-\frac{1}{R C s}\end{array}$ & Does not exist. \\
\hline \multicolumn{2}{|c|}{ Forward paths } & \multirow{2}{*}{$\Delta_{g}$} \\
\hline Paths from $\tilde{v}_{g}$ to $\tilde{v}_{o}$ & gain & \\
\hline$P_{g}: \tilde{v}_{g} \rightarrow s \tilde{i}_{L} \rightarrow \tilde{i}_{L} \rightarrow s \tilde{v}_{o} \rightarrow \tilde{v}_{o}$ & $P_{g}=\frac{\bar{D}_{1} \bar{D}_{2}}{L C s^{2}}$ & $\Delta_{g}=1$ \\
\hline \multicolumn{2}{|c|}{ Forward paths } & \multirow{2}{*}{$\Delta_{d}$} \\
\hline Paths from $\tilde{d}$ to $\tilde{v}_{o}$ & gain & \\
\hline $\begin{array}{c}P_{1 d}: d \rightarrow s \tilde{i}_{L} \rightarrow \tilde{i}_{L} \rightarrow s \tilde{v}_{o} \rightarrow \tilde{v}_{o} \\
P_{2 d}: d \rightarrow s \tilde{v}_{o} \rightarrow \tilde{v}_{o}\end{array}$ & $\begin{array}{c}P_{1 d}=\frac{\bar{V}_{g}}{L C s^{2}} \\
P_{2 d}=-\frac{\bar{D}_{1} \bar{V}_{g}}{R C \bar{D}_{2}^{2} s}\end{array}$ & $\begin{array}{l}\Delta_{d 1}=1 \\
\Delta_{d 2}=1\end{array}$ \\
\hline \multicolumn{3}{|c|}{ Transfer function from $\tilde{v}_{g}$ to $\tilde{v}_{o}$} \\
\hline$\frac{\tilde{v}_{o}}{\tilde{v}_{g}}=\sum \frac{P_{g} \Delta_{g}}{\Delta}=\frac{P_{g}}{1-L p_{1}-L p_{2}}=$ & & \\
\hline \multicolumn{3}{|c|}{ Transfer function from $\tilde{d}$ to $\tilde{v}_{o}$} \\
\hline$\frac{\tilde{v}_{o}}{d}=\sum \frac{P_{d} \Delta_{d}}{\Delta}=\frac{P_{1 d}+P_{2 d}}{1-L p_{1}-L p_{2}}==$ & & \\
\hline
\end{tabular}

$$
\begin{aligned}
& L \frac{d \bar{i}_{L}}{d t}=\bar{d} \bar{v}_{g}-\left(\bar{d}_{2}\right) \bar{v}_{o} \\
& C \frac{d \bar{v}_{o}}{d t}=-\frac{\bar{v}_{o}}{R}+\left(\bar{d}_{2}\right) \bar{i}_{L}
\end{aligned}
$$

Doing small signal linearization on averaged equations $\quad$ The simplified equations after linearization

$$
\begin{array}{c|c}
L s\left(\tilde{i}_{L}+\bar{I}_{L}\right)=\left(\bar{D}_{1}+d\right)\left(\tilde{v}_{g}+\bar{V}_{g}\right)-\left(\overline{D_{2}}-d\right)\left(\tilde{v}_{o}+\bar{V}_{o}\right) & L s \tilde{i}_{L}=\bar{D}_{1} \tilde{v}_{g}+\bar{V}_{g} d-\left(\bar{D}_{2}\right) \tilde{v}_{o}+\bar{V}_{o} d \\
C s\left(\tilde{v}_{o}+\bar{V}_{o}\right)=-\frac{\tilde{v}_{o}}{R}-\frac{\bar{V}_{o}}{R}+\left(\bar{D}_{2}-d\right)\left(\tilde{i}_{L}+\bar{I}_{L}\right) & C s \tilde{v}_{o}=-\frac{\tilde{v}_{o}}{R}+\left(\bar{D}_{2}\right) \tilde{i}_{L}-\bar{I}_{L} d \\
\hline \text { DC equations } & \text { Small signal ac equations } \\
\hline \bar{V}_{o}=\frac{\bar{D}_{1} \bar{V}_{g}}{\bar{D}_{2}} & s \tilde{i}_{L}=\frac{\bar{D}_{1}}{L} \tilde{v}_{g}+\frac{\bar{V}_{g}}{L \bar{D}_{2}} d-\frac{\bar{D}_{2}}{L} \tilde{v}_{o} \\
\bar{I}_{L}=\frac{\bar{V}_{o}}{R \bar{D}_{2}}=\frac{\bar{D}_{1} \bar{V}_{g}}{R \bar{D}_{2}^{2}} & s \tilde{v}_{o}=-\frac{\tilde{v}_{o}}{R C}+\frac{\bar{D}_{2}}{C} \tilde{i}_{L}-\frac{\bar{D}_{1} \bar{V}_{g}}{R C \bar{D}_{2}^{2}} d
\end{array}
$$

Table 2. Loop and path information of the signal flow graph and the obtained transfer functions 
Table 3. Achieved transfer functions for buck-boost converter in existence of the parasitic resistor of the inductor and without it

\begin{tabular}{|c|c|}
\hline $\begin{array}{c}\text { Transfer functions in existence of the parasitic resistor of the inductor } \\
\left(R_{L}\right)\end{array}$ & $\begin{array}{c}\text { Transfer functions in absence of the parasitic resistor of the inductor } \\
\left(R_{L}\right)\end{array}$ \\
\hline$\frac{\tilde{v}_{o}}{\tilde{v}_{g}}=\frac{\frac{D_{1}}{D_{2}}}{\left(\frac{L C}{D_{2}^{2}}\right) s^{2}+\left(\frac{\left.R_{L} C+\frac{L}{R}\right)}{D_{2}^{2}}\right) s+\left(1+\frac{\left(\frac{R_{L}}{R}\right)}{D_{2}^{2}}\right)}$ & $\frac{\tilde{v}_{o}}{\tilde{v}_{g}}=\frac{R D_{1} D_{2}}{R L C s^{2}+L s+R D_{2}^{2}}$ \\
$\frac{\tilde{v}_{o}}{\tilde{d}}=\frac{V_{g}+V_{o}-\left(\frac{L s+R_{L}}{D_{2}}\right) I_{L}}{\left(\left(\frac{L C}{D_{2}^{2}}\right) s^{2}+\left(\frac{\left.R_{L} C+\frac{L}{R}\right)}{D_{2}^{2}}\right) s+\left(1+\frac{\left(\frac{R_{L}}{R}\right)}{D_{2}^{2}}\right)\right)}$ & $\frac{\tilde{v}_{o}}{\tilde{d}}=\frac{V_{g}\left(R-\frac{D_{1} L}{D_{2}{ }^{2}} s\right)}{R L C s^{2}+L s+R D_{2}{ }^{2}}$ \\
\end{tabular}

Table 4. The buck-boost converter component values

\begin{tabular}{|c|c|}
\hline$L$ & $0.43 m H$ \\
$C$ & $33 \mu F$ \\
$R$ & $10 \Omega$ \\
$R_{L}$ & $0.25 \Omega$ \\
$V_{g}$ & $15 v$ \\
\hline
\end{tabular}

Table 5. Information obtained from poles and zeros of the transfer functions of the model

\begin{tabular}{|c|c|c|c|}
\hline \multirow{2}{*}{} & The transfer function $\frac{\tilde{v}_{O}}{\tilde{v}_{g}}$ & \multicolumn{2}{|c|}{ The transfer function $\frac{\tilde{v}_{O}}{\tilde{d}}$} \\
\cline { 2 - 4 } & Poles & Poles & Zero \\
\hline Value & $(-1.81 \pm 5.27 i) \times 10^{3}$ & $(-1.81 \pm 5.27 i) \times 10^{3}$ & $2.77 \times 10^{4}$ \\
Damping & 0.324 & 0.324 & -1 \\
Overshoot (\%) & 34.1 & 34.1 & 0 \\
Frequency (rad/s) & $5.57 \times 10^{3}$ & $5.57 \times 10^{3}$ & $2.77 \times 10^{4}$ \\
\hline
\end{tabular}

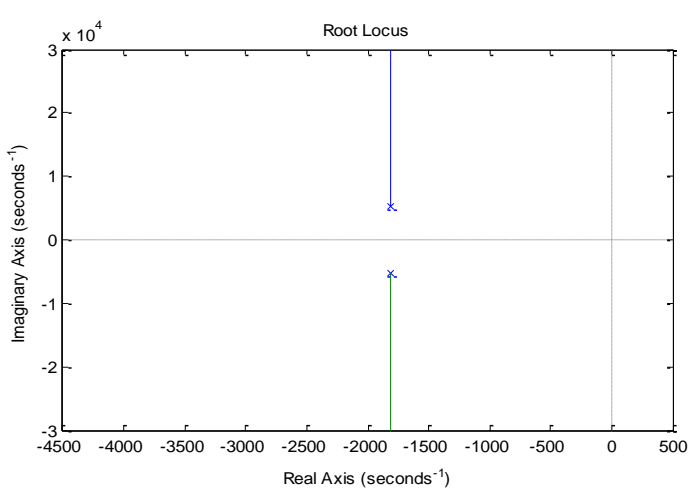

(a)

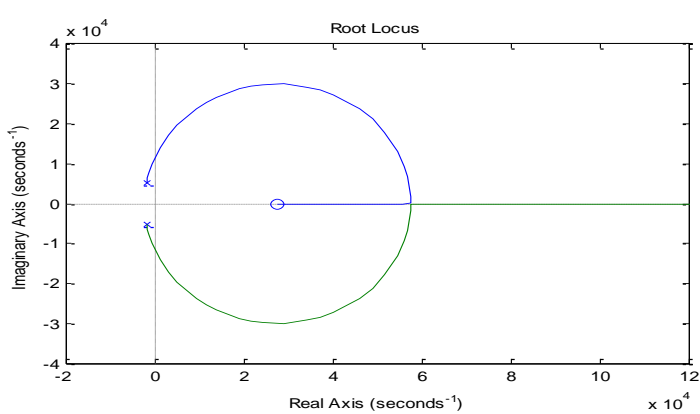

(b)

Figure 3. Root locus diagram; (a) $\tilde{v}_{o} / \tilde{v}_{g} ;$ (b) $\tilde{v}_{o} / \tilde{d}$ with $R_{L}=0.25 \Omega$

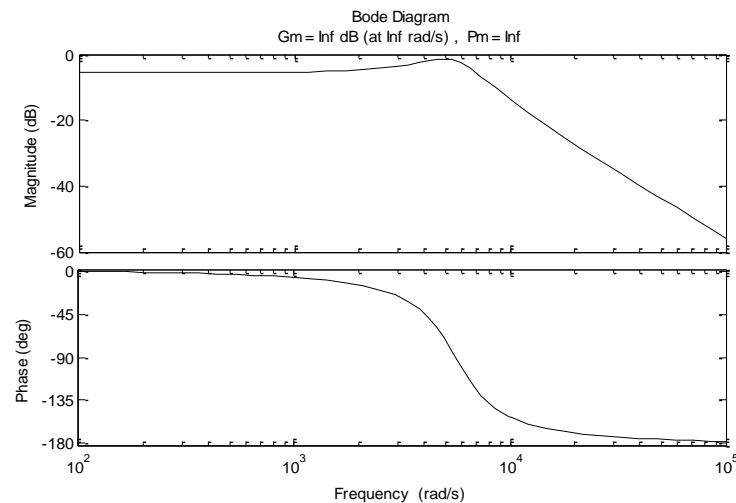

(a)

Bode Diagram

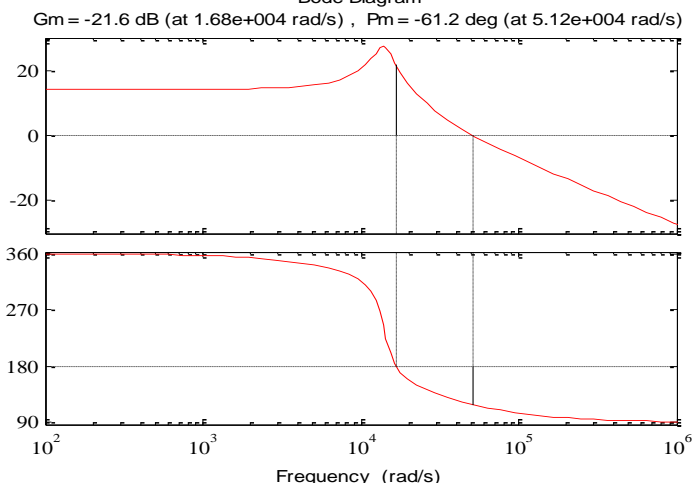

(b)

Figure 4. Bode diagrams; (a) $\tilde{v}_{o} / \tilde{v}_{g}$; (b) $\tilde{v}_{o} / \tilde{d}$ 


\section{The Proposed Model-Based Full State Feedback Controller Configuration}

Fig. 5 shows the step response of the system. This figure shows that the open-loop system does not reject disturbances on the input voltage and cannot regulate the output voltage.

The control action covers pole placement by using full state feedback. The whole configuration of the controller beside the controlled system is plotted in Fig. 6. In order to control the converter based on the previously extracted model, the state space form of the controlled transfer function is calculated. In Fig. 6, $A, B, B_{d}$, and $C$ are the state space matrixes of the system and $K$ is the feedback gain. In the whole design process, the control input to the buck-boost converter is changed the duty cycle used to turn on and off the switch.

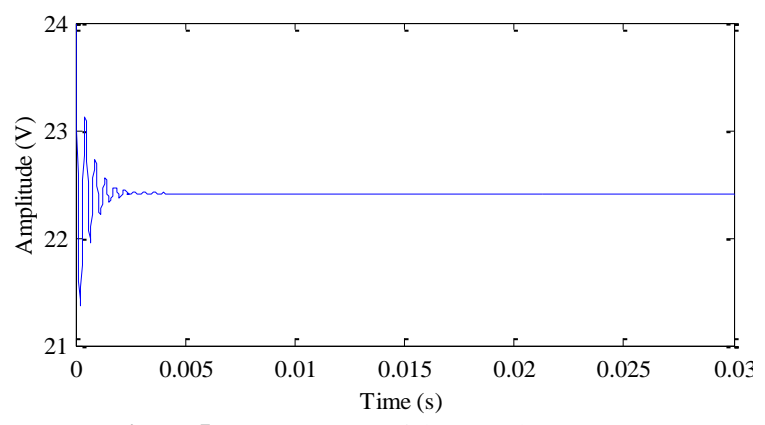

Figure 5. Step response of the open loop system

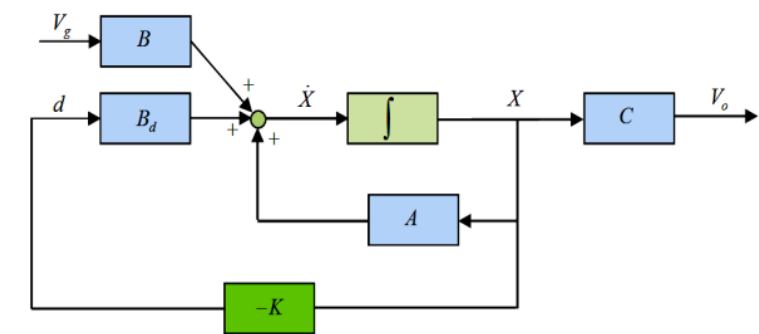

Figure 6. The whole system configuration with the full state feedback controller

\section{Pole Placement By Using Ackermann's Formula}

After checking the system controllability and observability by using the related matrixes, now the pole placement is done. Ackermann's formula is used for single-input, single-output (SISO) systems like the buck-boost converter. Ackermann's formula is:

$$
K=\left[\begin{array}{lll}
0 & 0 & \ldots 1
\end{array}\right] M_{c}^{-1}\left(A^{n}+\alpha_{1} A^{n-1}+\ldots+\alpha_{n-1} A+\alpha_{n} I\right)
$$

In above equation, $M_{c}$ is the controllability matrix and $n$ is the order of the system.

As the controllability matrix is a full rank matrix, the system is fully controllable. About the observability issue, the system turns fully observable. Then any proper pole placement method can be used to get the desired behaviour of the system.

\subsection{Pole Placement}

In a completely controllable system in which all the states are accessible, in order to place the poles at any desired location, feedback of all of the states through a gain matrix can be employed. The control law for such a full state feedback is:
$u=-K X$

The matrix $K$ is a gain matrix used to place the poles of the system at any desired locations.

\subsection{Regulating the Converter Output Voltage with State Feedback Compensator}

In order to achieve a stable control in all operating ranges of the converter system by using the obtained model, the poles should be placed in a left location. One problem with this matter is selecting desirable pole locations. Two main methods exist for this issue [16]:

1) Choosing pole locations in such a way that a dominant complex pole pair exists. This technique is used for designing tracking systems, for which the transient time domain characteristics (rise time, overshoot, settling time ...) can bring out the desired dominant pole locations.

2) Choosing pole locations specified to give a prototype time domain response, e.g., filter pole locations.

The second method is used for pole placement with full state feedback control. In [16] assigning the system poles to prototype locations that minimize a cost function is discussed. This method is known as the integral of the time-weighted absolute error (ITAE) to an input signal as follows

$$
J_{\text {ITAE }}=\int_{0}^{\infty} t|e(t)| d t
$$

Since the aim of the control system is to regulate the buck-boost converter output voltage regarding the input voltage disturbances, then the desired output is rejection of disturbance deviations from the nominal operating point (the set point for the steady state error). The error between the desired output and the system output is defined as $e(t)=r(t)-y(t)$. As $r(t)=0$ for all time, then the error becomes equal to $y(t)$.

By using Matlab software, the frequency normalized pole locations for ITAE response for the buck-boost converter system which is a second order one, becomes $\omega^{2}[-0.7071 \pm j 0.7071]$. Then the value of $\omega$ should be found in a way that places the poles to get the desired time domain response. In each iteration, the steady state error is used as a measurement metric and it should be kept $1 \%$ of the desired output voltage $24 \mathrm{~V}$.

The step response of the plant with the designed full state feedback controller is shown in Fig. 7.

From the $0.24 \mathrm{~V}$ steady-state errors to the $1 \mathrm{~V}$ step disturbance in input voltage, it is obvious that the disturbance is rejected and this controller behaves very desirably in time-domain. Checking the root-locus diagram shown in Fig. 8 depicts that the system has become a stale and minimum phase system. Fig. 9 shows the frequency domain responses of the regulated system.

Calculations bring out the gain margin as infinity and a phase margin of 69.1 degree, which are favourite frequency-domain response characteristics. Then, by applying the proposed controller the converter system becomes stable and regulated.

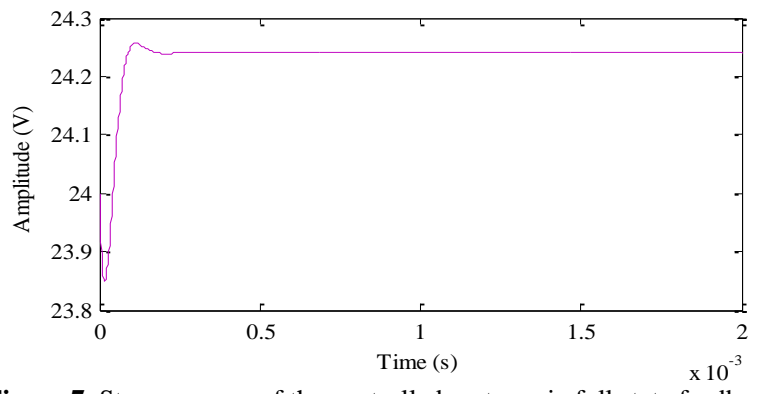

Figure 7. Step response of the controlled system via full state feedback 


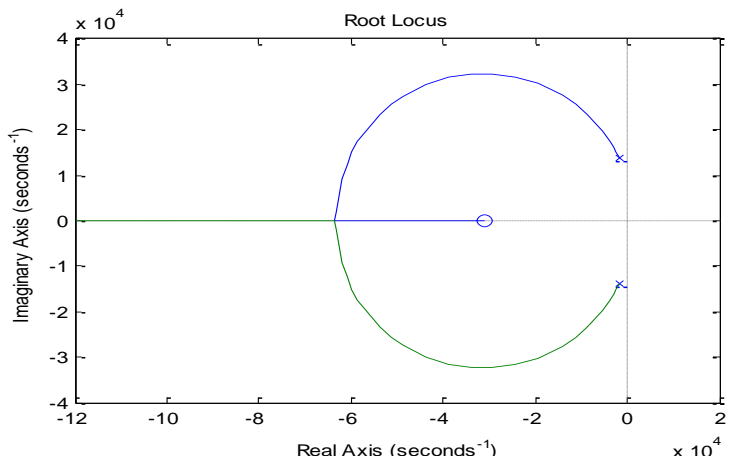

Figure 8. Root- locus diagram of $\tilde{v}_{o} / \tilde{d}$ after applying the designed feedback regulator

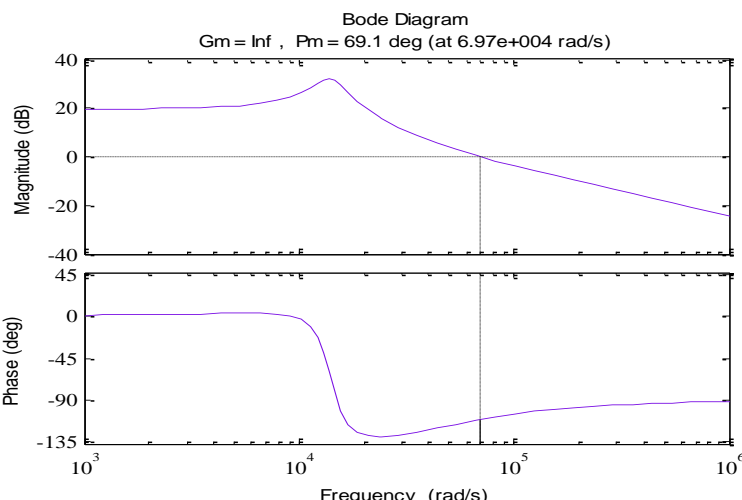

Figure 9. Bode diagrams of $\tilde{v}_{o} / \tilde{d}$ after applying the designed feedback regulator

\section{Conclusions}

In this paper, the application of signal flow graph technique and Mason gain formula for extracting associated transfer functions and modelling a buck-boost dc-dc converter is investigated. Transfer functions from input to output and control to output have been obtained. Employing the obtained functions, stability analysis and deciding about the controller design is performed. Finally, the efficiency of the state space feedback controller as a model based technique to design a proper control system is shown. The proposed control system employs the feedback controller with a special adjustment for proper setting of the control responses.

\section{References}

[1] M. H. Rashid, "Power electronics handbook," Elsevier Academic Press, 2001.

[2] X. Wei, K.M. Tsang, and W.L. Chan, "DC/DC buck converter using internal model control," Journal of Electr. Power Compo. Sys., vol. 37, no. 3, pp. 320-330, 2009.
[3] K.P. Yalamanchili, M. Ferdowsi, Sh. Lu, P. Xiao, and K. Corzine, "Derivation of double-input dc-dc power electronic converters," Journal of Electr. Power Compo. Sys., vol. 39, no. 5, pp. 478-490, 2011.

[4] F.L. Luo and H. Ye, "Mathematical modeling for dc-dc converters," IEEE Trans. Power Electron., vol. 22, no. 1, 2007.

[5] R. Priewasser, "Modeling, control and digital implementation of dc-dc converters under variable switching frequency operation," Ph.D. Thesis, Klagenfurt university, 2012.

[6] S. Kapat, "Control methods for improving the performance of dc-dc converters," Ph.D. Thesis, Kharagpur, India, Jul. 2009.

[7] L.K. Wong and T.K. Man, "Small signal modeling of open-loop SEPIC converters," IET Power Electron., vol. 3, no. 6, pp. 858-868, May 2010.

[8] H. Mashinchi Mahery and E. Babaei, "Mathematical modeling of buck-boost dc-dc converter and investigation of converter elements on transient and steady state responses," Electr. Power and Ener. Sys., vol. 44, pp. 949963, 2013.

[9] E. Babaei and H. Mashinchi Maheri, "Analytical solution for steady and transient states of buck dc-dc converter in CCM," Arab. Journal Sci. and Eng., vol. 38, no. 12, pp. 3383-3397, 2013.

[10] M. Veerachary, "General rules for signal flow graph modeling and analysis of dc-dc converters," IEEE Trans. Aerospace Electron. Sys., vol. 40, no. 1, pp. 259-271, Jan. 2004.

[11] M. Veerachary, "Signal flow graph modeling of cascade boost converters," in Proc. PESC, 2003, vol. 2, pp. 606609.

[12] M. Veerachary, "Signal flow graph modeling of multi-state boost dc-dc converters," in Proc. IEE -Electr. Power Appl., vol. 151, no. 5, pp. 583-589, Sep. 2004.

[13] K. Smedley and S. Cuk, "Switching flow-graph nonlinear modeling technique," IEEE Trans. Power Electron., vol. 9 , no. 4, pp. 1-1, Jul. 1994.

[14] M. Veerachary, "Analysis of fourth-order dc-dc converters: A flow graph approach," IEEE Trans. Ind. Electron., vol. 55, no. 1, pp. 133-141, Jan. 2008.

[15] M. Veerachary, T. Senjyu and K. Uezato, "Signal flow graph nonlinear modeling analysis of IDB converter," in Proc. ISIE, 2001, Pusan, Korea, pp. 1066-1070.

[16] R. T. Stefani, B. Shahian, C. J. Savant, Jr., and G. H. Hostetter, "Design of feedback control systems," 4th ed. New York, NY: Oxford University Press, 2002. 